\title{
Imperial College STANDARD FILTER APPROXIMATIONS FOR LOW POWER London CONTINUOUS WAVELET TRANSFORMS
}

Alexander J. Casson and Esther Rodriguez-Villegas

Circuits and systems research group, Electrical and Electronic Engineering Department, Imperial College London Email: \{acasson, e.rodriguez\}@imperial.ac.uk

\section{Abstract}

- Low power Continuous Wavelet Transform (CWT) circuits are essential for use in wearable, long term physiological monitoring systems.

- Present analogue CWT circuits implement the CWT as a bandpass filter and rely on taking a mathematical approximation of the wanted mother wavelet function.

- We present an alternative approximation technique based upon the use of standard filter approximations: Butterworth, Chebyshev and Bessel bandpass filters.

\section{Low power CWT circuits}

- At a single analysis scale the CWT is a bandpass filtering operation [1] - The required bandpass filter must have an impulse response function:

$$
h(t)=\frac{1}{\sqrt{a}} \psi\left(\frac{-t}{a}\right)
$$

- where $a$ is the analysis scale and $\psi(t)$ is the mother wavelet function.

- The bandpass filter can be in either the analogue or digital domain:

- Analogue approaches are popular as they have very low power

consumption, typically in the order of nano-Watts [1, 2].

- However, analogue filters can only implement an approximation of $h(t)$.

\section{Current mathematical approximation}

- Using the Mexican hat mother wavelet as an example, the ideal $h(t)$ impulse response is:

- However this is symmetrical around $t=0$.

- It is non-causal and cannot be implemented directly by a low power analogue filter.

- Thus [1] presented the LPCWT as a technique to approximate the ideal $h(t)$ giving an $h(t)$ function that can be implemented.

- If $H(j \omega)$ is the Fourier Transform of $h(t)$ and $s=j \omega$ :

Ideal Mexican hat LPCWT approximation

$$
H(s)=\frac{-\pi^{1 / 4} \sqrt{8 a^{5} / 3} s^{2}}{e^{-a^{2} s^{2} / 2}} \longrightarrow H(s)=\frac{-\pi^{1 / 4} \sqrt{8 a^{5} / 3} s^{2}}{1+s T+\left(\frac{T^{2}}{2}-\frac{a^{2}}{2}\right) s^{2}+\left(\frac{T^{3}}{6}-\frac{T a^{2}}{2}\right) s^{3}+\cdots}
$$

- where a delay $T$ has been introduced and the denominator has been expanded as a truncated Maclaurin series.

- The resulting impulse response function is:

\section{Demonstration of operation}

- To demonstrate the utility of the proposed approximations we use them to perform a CWT analysis of ECG data.

- The ECG record is taken from the MIT-

BIT noise stress database [3]:

- It is measured ambulatory ECG corrupted by baseline wander, muscle artefact and electrode motion artefact.

- For each approximation method we use 98 bandpass filters in parallel:

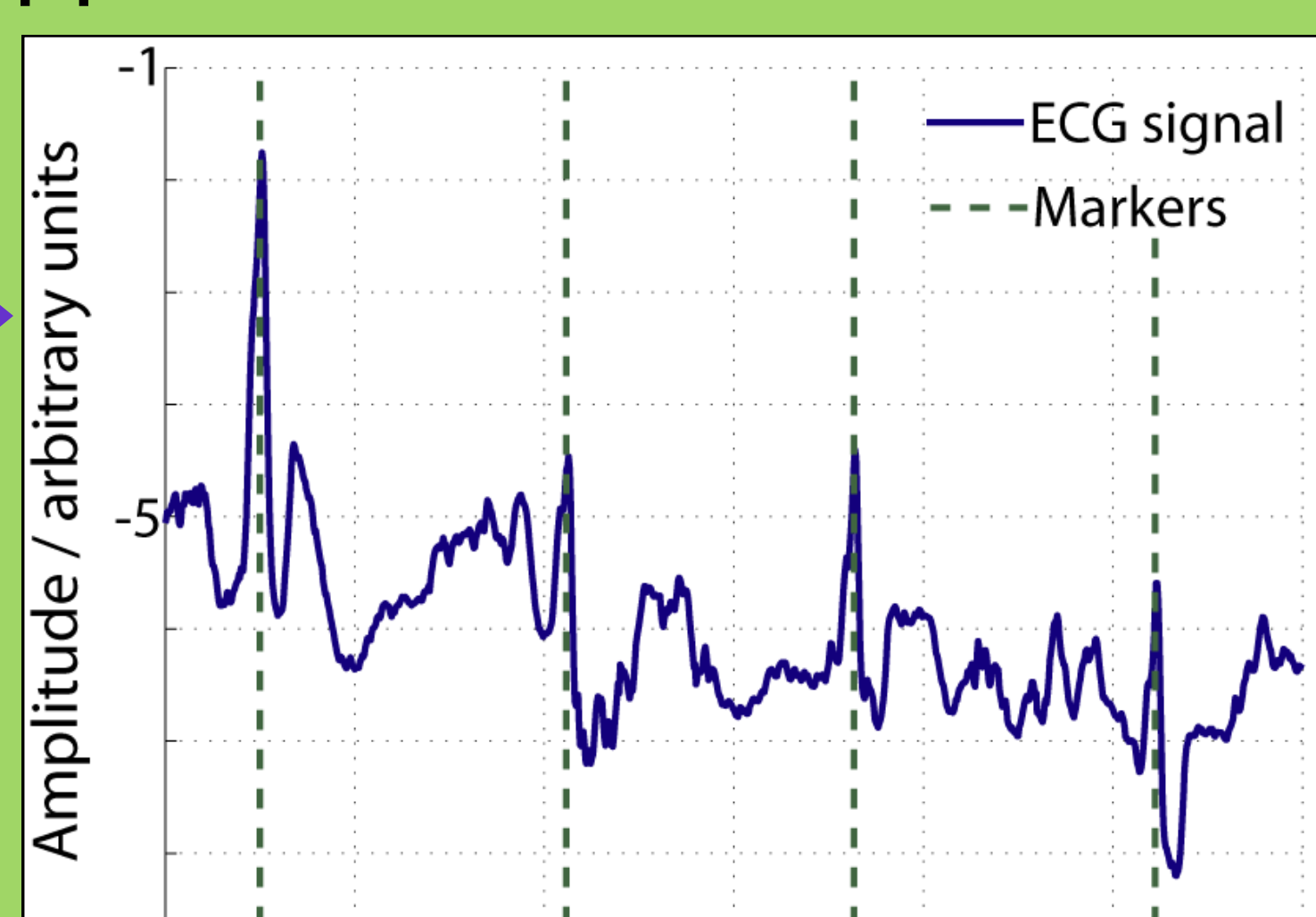

- For correct wavelet operation the gain of each

filter changes with the centre frequency $\omega_{\mathrm{c}}$ as $\Rightarrow H(s) \rightarrow \sqrt{\frac{3.63}{\omega_{c}}} \times H(s)$

- Centre frequencies are logarithmically spaced.

- Below, colour represents the absolute output signal from each filter versus time and the filter centre frequency for the ECG signal above:
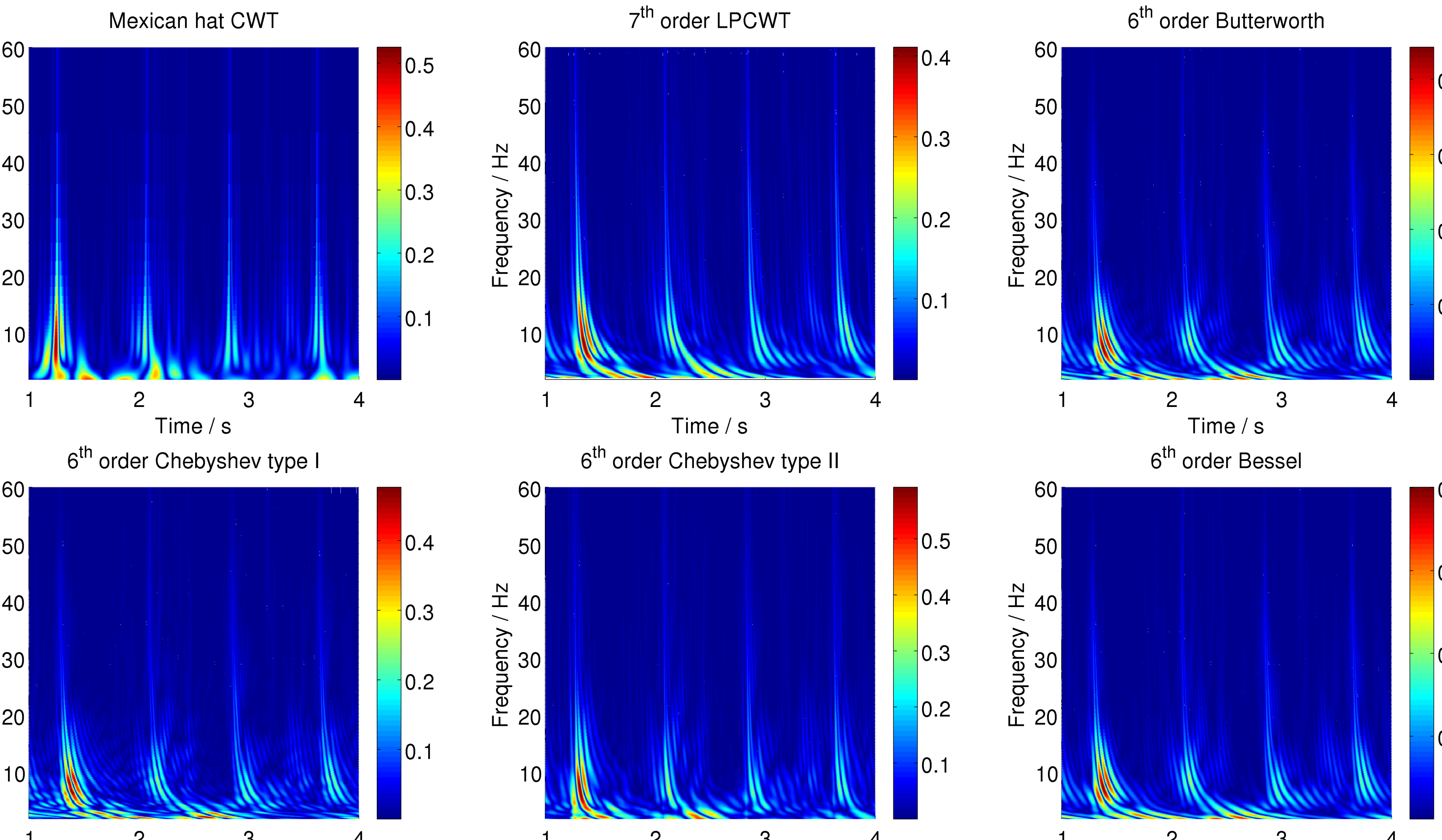

- Compared to the ideal Mexican hat CWT all the approximations have a delay present at low frequencies.

- Nevertheless, similar ECG information is extracted in all cases.

- The Mexican hat approximations proposed here thus work, and provide alternatives to the current mathematical approach.

- Furthermore, there is a large body of work on the design of circuits implementing Butterworth, Chebyshev and Bessel transfer functions, easing the circuit level design required.
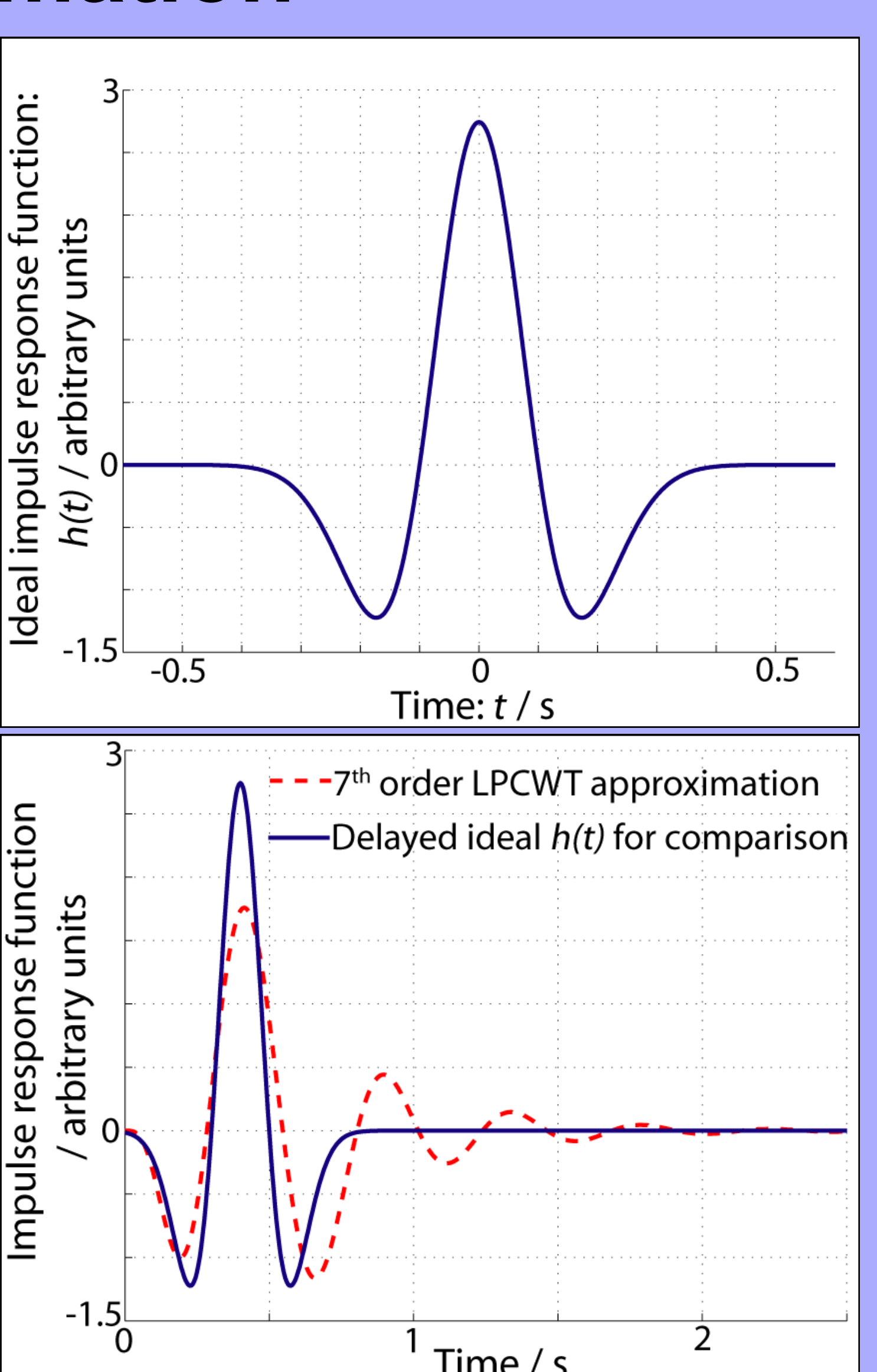

\section{New standard filter approximation}

-We present a new approximation procedure,

increasing the range of options available.

- Filters with Butterworth, Chebyshev and Besse

transfer functions are used in many analogue systems:

- These filter functions are known as the standard filter approximations.

- However, such filters are normally designed in the frequency domain:

- The filters are designed to provide a set attenuation at a certain frequency.

- Instead we look at the time domain

- What does the impulse response of each filter type look like?

- Can the impulse responses be tailored to look like the Mexican hat impulse response?

- Bandpass transfer functions are generated in MATLAB and parameters adjusted to get the impulse responses to match the Mexican hat shape:

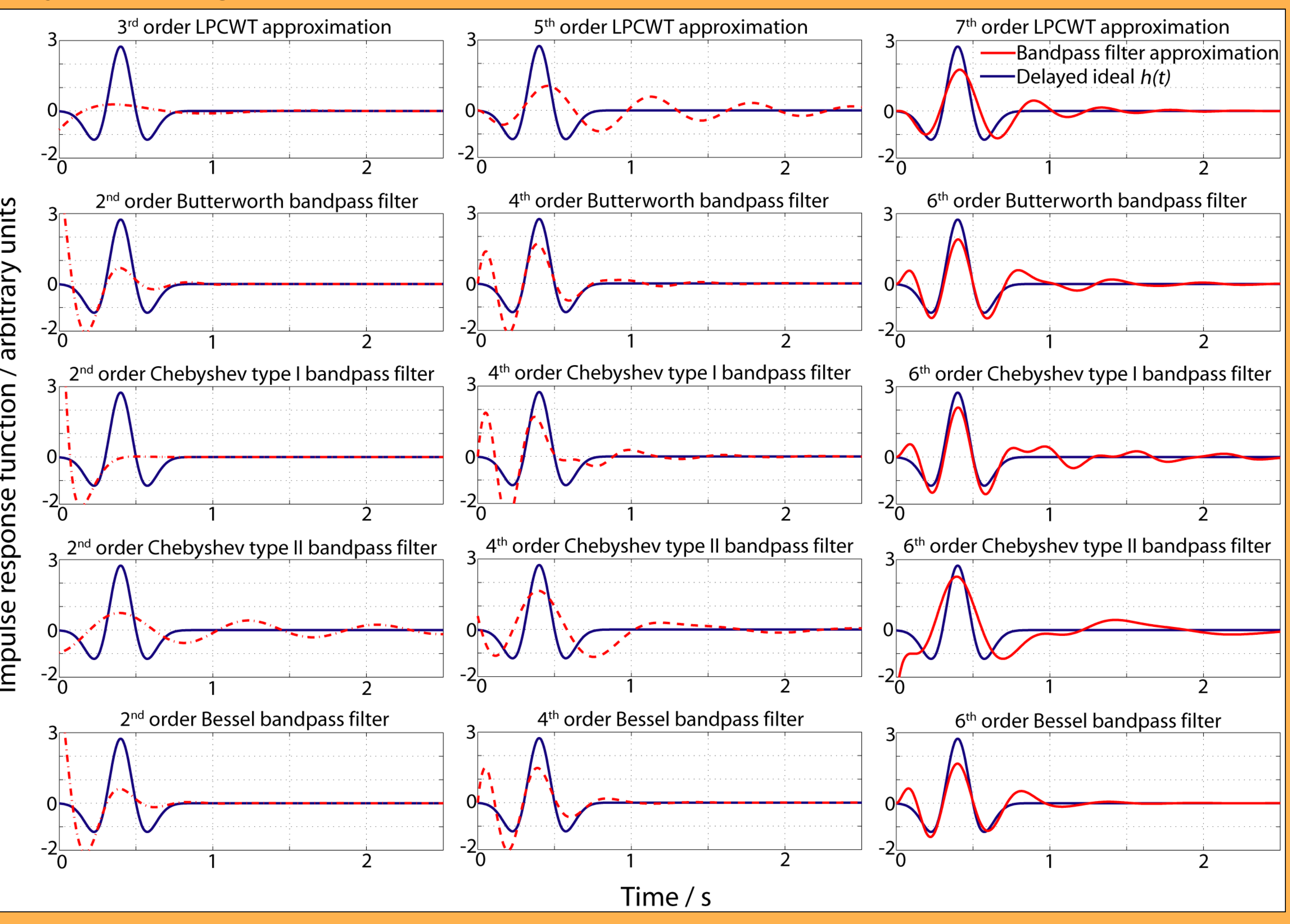

- For comparison, the more traditional frequency domain view:

- As the filter order increases, better approximations to the Mexican hat impulse response are achieved.

- Thus Butterworth, Chebyshev and Bessel bandpass filters can approximate the wanted $h(t)$ function.

- The parameters used to achieve this:

\begin{tabular}{ll}
\hline Filter & Parameters \\
\hline Butterworth & Centre freq. $=15 \mathrm{rads}^{-1}, Q=1.5$ \\
Chebyshev type I & Centre freq. $=15 \mathrm{rads}^{-1}, Q=1.35$ \\
& Ripple $(\varepsilon)=0.8$ \\
Chebyshev type II & $\begin{array}{l}\text { Centre freq. }=7.5 \mathrm{rads}^{-1}, \mathrm{Q}=0.55 \\
\text { Ripple }(\varepsilon)=20\end{array}$ \\
Bessel & Centre freq. $=15 \mathrm{rads}^{-1}, \mathrm{Q}=1.35$
\end{tabular}

- By varying these parameters other mother wavelets can also be approximated.

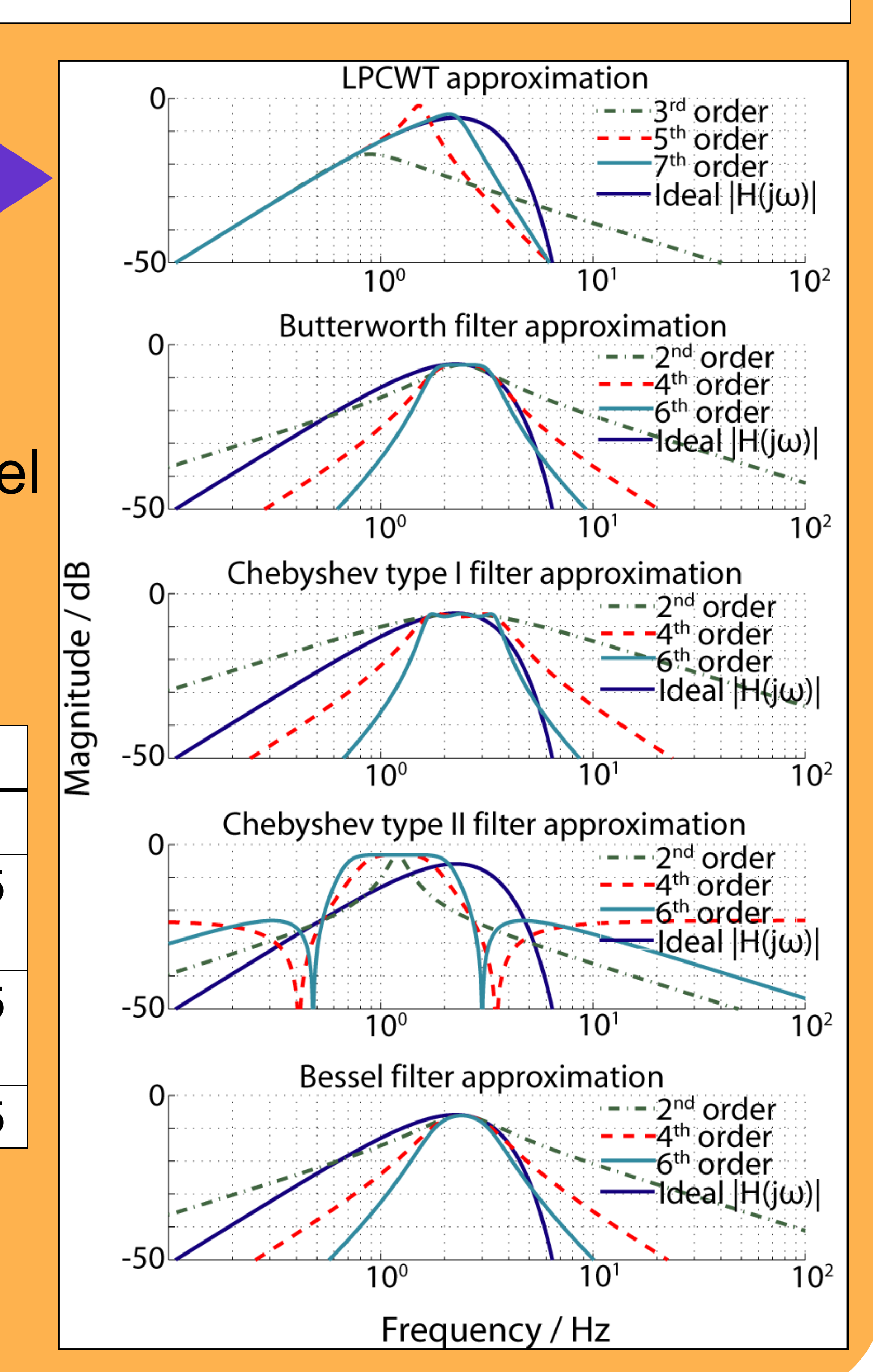

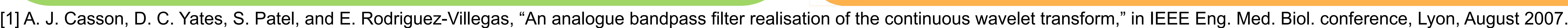
[2] S. A. P. Haddad and W. A. Serdijn, Ultra low-power biomedical signal processing: An analog wavelet approach for pacemakers. Springer, 2009.

[3] PhysioBank. (2010) Home page. [Online]. Available: http://www.physionet.org/physiobank/.

The research leading to these results has received funding from the European Research Council under the European Community's $7^{\text {th }}$ Framework Programme / ERC grant agreement no. 239749. 